\title{
Comparison of outcomes in patients with methicillin-susceptible Staphylococcus aureus (MSSA) bacteremia who are treated with $\beta$-lactam vs vancomycin empiric therapy: a retrospective cohort study
}

\author{
Davie Wong ${ }^{1 *}$, Titus Wong ${ }^{2,3}$, Marc Romney ${ }^{2,4}$ and Victor Leung ${ }^{2,4}$
}

\begin{abstract}
Background: Prior studies suggested that vancomycin may be inferior to $\beta$-lactams for the empiric treatment of methicillin-susceptible S. aureus (MSSA) bacteremia. We assessed whether empiric therapy with $\beta$-lactams compared to vancomycin was associated with differences in clinical outcomes in patients with MSSA bacteremia.

Methods: We conducted a retrospective cohort study of adult inpatients with their first episode of MSSA bacteremia at two tertiary care hospitals in Vancouver, Canada, between 2007 and 2014. Exposure was either empiric $\beta$-lactam or vancomycin therapy. All patients received definitive treatment with cloxacillin or cefazolin. The primary outcome was 28-day mortality. Secondary outcomes were 90-day mortality, recurrent infection at 6 months, duration of bacteremia and hospital length-of-stay. Outcomes were adjusted using multivariable logistic regression.

Results: Of 814 patients identified, 400 met inclusion criteria $(\beta$-lactam $=200$, vancomycin $=200)$. Overall 28-day mortality was $8.5 \%(n=34)$. There were more cases of infective endocarditis in the $\beta$-lactam than in the vancomycin group [45 (22.5\%) vs $23(11.5 \%), p<0.01]$. Adjusted mortality at 28 days was similar between the two groups (OR: 1.14; $95 \%$ Cl: 0.49-2.64). No differences in secondary outcomes were observed. Transition to cloxacillin or cefazolin occurred within a median of $67.8 \mathrm{~h}$ in the vancomycin group.

Conclusions: Empiric therapy with $\beta$-lactams was not associated with differences in all-cause mortality, recurrent infection, microbiological cure or hospital length-of-stay compared to vancomycin. Vancomycin monotherapy may be appropriate for the empiric treatment of MSSA bacteremia if definitive therapy with cloxacillin or cefazolin can be initiated within 3 days.
\end{abstract}

Keywords: Staphylococcus aureus, Bacteremia, Empiric, Therapy, Beta-lactam, Vancomycin

\section{Background}

Staphylococcus aureus is the leading cause of bacteremia and carries a mortality of $20-30 \%$ in the 21 st century $[1,2]$. Empiric vancomycin is commonly prescribed for patients with $S$. aureus bacteremia (SAB) to cover methicillin-resistant $S$. aureus (MRSA) as up to 50-60\%

\footnotetext{
* Correspondence: wongdavi@mail.ubc.ca

${ }^{1}$ PGY-V Infectious Diseases Residency Training Program, University of British Columbia, Vancouver General Hospital, D 452 Heather Pavilion, 2733 Heather Street, Vancouver, BC V5Z 1 M9, Canada

Full list of author information is available at the end of the article
}

of bloodstream isolates are methicillin-resistant at some centres [3-8]. However, vancomycin is inferior to semisynthetic anti-Staphylococcal penicillins (e.g., cloxacillin) and first generation cephalosporins (e.g., cefazolin) for the definitive treatment of methicillin-susceptible $S$. aureus (MSSA) bacteremia [9-11]. Cloxacillin and cefazolin are considered the optimal agents against MSSA and both are equally efficacious in treating MSSA bacteremia [10]. Vancomycin is associated with higher rates of infection-related mortality, re-infection and bacteriologic failure compared to cloxacillin or cefazolin in the 
definitive treatment of MSSA bacteremia [9, 12-15]. Whether vancomycin is inferior to $\beta$-lactams for empiric therapy remains to be fully elucidated. Early studies suggested that empiric vancomycin was associated with worse outcomes compared to empiric $\beta$-lactam therapy $[3,16,17]$, but more recent data did not demonstrate any differences in outcomes [15]. Although controversial, some experts recommend the addition of a $\beta$-lactam agent to vancomycin during empiric treatment to ensure optimal coverage for MSSA for patients at the highest risk of morbidity and mortality from SAB [18]. Major limitations of previous studies were failure to control for the definitive therapy prescribed when comparing empiric regimens and neglecting to specify the empiric $\beta$-lactams used $[3,15-17]$. We assessed if empiric $\beta$-lactam compared to vancomycin was associated with differences in survival, recurrent infection and microbiological cure in patients with MSSA bacteremia who received definitive therapy with cloxacillin or cefazolin.

\section{Methods \\ Patients}

We performed a retrospective cohort study of adult inpatients diagnosed with their first episode of MSSA bacteremia at two tertiary care hospitals in Vancouver, Canada, between January 2007 and December 2014, inclusive. Consecutive patients were included if they had MSSA bacteremia and either cloxacillin or cefazolin was prescribed for definitive therapy (penicillin was an acceptable alternative if the isolate was proven to be susceptible). Patients were excluded if there was missing data for 28-day mortality, no empiric therapy was administered, death occurred within $24 \mathrm{~h}$ following diagnosis of bacteremia, or polymicrobial bacteremia. Patients were stratified based on empiric treatment with $\beta$-lactams or vancomycin. The $\beta$-lactam group received one or more of cloxacillin, cefazolin, $\beta$-lactam/ $\beta$-lactamase inhibitor combination, a third generation cephalosporin or a carbapenem, with or without vancomycin. In both groups, other antimicrobials may have been prescribed during empiric and definitive therapy.

\section{Definitions}

Bacteremia was defined as the isolation of MSSA from one or more blood culture bottles. Bacteremia identified within $72 \mathrm{~h}$ of hospital admission was considered communityonset, while bacteremia diagnosed after more than $72 \mathrm{~h}$ of hospital admission was deemed hospital-onset. Immunocompromised state was present if any of the following were described: neutropenia $\left(\leq 1.5 \times 10^{9} / \mathrm{L}\right)$, congenital immune deficiencies, or use of immunosuppressants (TNF- $\alpha$ inhibitors, prednisone $\geq 10 \mathrm{mg} /$ day or its equivalent, cancer chemotherapy, methotrexate, cyclophosphamide, mycophenolate mofetil, calcineurin inhibitors, mTOR inhibitors, azathioprine and any other drug generally considered to significantly weaken the immune system). Definite infective endocarditis was diagnosed using the modified Duke criteria [19]. The source of bacteremia was either stated explicitly or inferred as the most likely source based on available clinical data and microbiological results. Metastatic complications included infections that occurred distant from the presumed primary source such as septic emboli, mycotic aneurysms, osteoarticular infections, and distant abscesses. Surgical source control included only procedures performed in the operating theatre. Empiric therapy began with the first dose of empiric antibiotics and ended with the start of definitive therapy. Definitive therapy began when antimicrobial susceptibilities were known and one of the following treatments was prescribed: 1) cloxacillin or cefazolin 2) discontinuation of other empiric antibiotics for patients already on cloxacillin or cefazolin empirically, or 3) continuation of empiric cloxacillin or cefazolin. Definitive therapy ended when cloxacillin or cefazolin was stopped. Time to receipt of antibiotics was measured from the time of obtaining the first positive blood culture to the time of the first dose of antibiotic. If a patient was already on antibiotics at the time of the first positive blood culture, the time to receipt of antibiotics was zero. We calculated the hours of empiric $\beta$-lactam exposure based on start and stop dates, start and stop times, and dosing frequency.

\section{Outcomes}

Our primary outcome was 28-day all-cause in-hospital mortality. Secondary outcomes were 90-day all-cause inhospital mortality, recurrent infection at 6 months, duration of bacteremia and hospital length-of-stay (LOS). Time to mortality was measured from the date of the first positive blood culture to the date of death. Recurrent infection occurred when MSSA bacteremia was diagnosed again following completion of a treatment course for the previous episode of MSSA bacteremia. Duration of bacteremia was the time difference between the first positive blood culture and the first negative blood culture. Patients without follow-up blood cultures were excluded from the analysis for duration of bacteremia. Hospital LOS was measured from the date of admission to the date of discharge.

\section{Data extraction}

Patients with MSSA bacteremia were extracted from the medical microbiology laboratory information systems and medical records were reviewed. A single reviewer collected data on patient demographics and comorbidities, blood culture results and antimicrobial therapy from electronic and paper databases. 


\section{Statistical analysis}

Our predicted mortality difference between the $\beta$-lactam and vancomycin group was $15 \%$ based on a previous study [17]. We estimated a sample size of 200 for each group to capture a $15 \%$ difference in mortality with $80 \%$ power at a two-tailed alpha level of 0.05. Baseline categorical variables were described as counts and percentages, and differences between groups were assessed with chi-square or Fisher's exact tests. Continuous variables were presented as means and standard deviations, or medians and interquartile range. Differences between groups were assessed using parametric $t$-tests or nonparametric Mann-Whitney- $U$ tests, as appropriate. Logistic regression methods were used to model the odds ratio of death and recurrent infection in the $\beta$-lactam compared to vancomycin group. Linear regression model was conducted for hospital LOS and duration of bacteremia. The two outcomes were log-transformed in the analysis to improve normality of the distribution of residuals. All models were adjusted for pre-specified confounding variables including age, sex, age-adjusted Charlson-comorbidity index [20], Pitt bacteremia score, infectious diseases consultation, infective endocarditis and time to receipt of empiric antibiotics. These factors have been shown to affect mortality in patients with SAB [1]. The duration of bacteremia was further adjusted for surgical source control. All analyses were performed using the SAS 9.4 software.

\section{Results}

We identified 814 patients with MSSA bacteremia between January 2007 and December 2014, inclusive (Fig. 1). We excluded 414 patients primarily because $60.4 \%$ did not receive cloxacillin or cefazolin for definitive therapy.
These patients either remained on broad-spectrum antimicrobials or received vancomycin for definitive therapy due to suspected or confirmed penicillin allergy. Another $22.9 \%$ were not started on empiric therapy. Our cohort consisted of $64.5 \%$ males and $82.8 \%$ of patients had community-onset bacteremia (Table 1). Infectious diseases consultation was obtained in most cases (70.5 \%), but was higher in the $\beta$-lactam than in the vancomycin group (75 \% vs $66 \%, p=0.05$ ). The most common sources of bacteremia were unknown (26\%), injection drug use (21.8\%), peripheral or central venous catheters (15.3\%), and skin and soft tissue infections (15\%). Infective endocarditis was diagnosed more frequently in the $\beta$-lactam than in the vancomycin group $(22.5 \%$ vs $11.5 \%, p<0.01)$. The prevalence of infective endocarditis was $17.0 \%$. The $\beta$-lactam group experienced more metastatic complications (36.5\% vs $26.5 \%, p=0.03)$ and underwent surgical source control more frequently $(21.5 \%$ vs $13 \%, p=0.02)$ compared to the vancomycin group.

The most common empiric antimicrobials prescribed in the $\beta$-lactam group were vancomycin (76.5\%), cloxacillin or cefazolin $(69 \%), 3^{\text {rd }}$ generation cephalosporins (40\%) and piperacillin-tazobactam (34.5\%). The use of multiple $\beta$-lactam antibiotics reflects changes made during empiric therapy. Among the subgroup of patients who received combination therapy with $\beta$-lactam plus vancomycin (153/200), cloxacillin or cefazolin (62.1\%), 3rd generation cephalosporins $(47.7 \%)$ and piperacillintazobactam $(42.5 \%)$ were the most common empiric $\beta$ lactams prescribed (Table 2). Cloxacillin or cefazolin $(91.5 \%)$ was the predominant empiric $\beta$-lactam used in the monotherapy subgroup. Initiation of cloxacillin or cefazolin was delayed in the combination subgroup compared to the $\beta$-lactam monotherapy subgroup (median

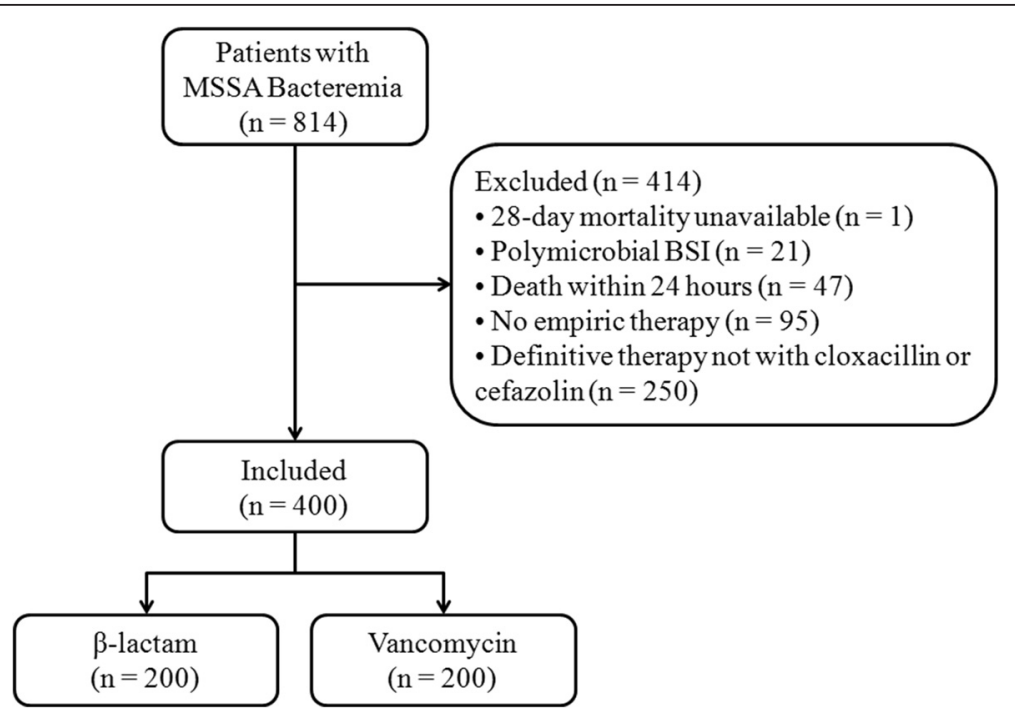

Fig. 1 Patient enrollment process. Abbreviations: MSSA methicillin-susceptible S. aureus, BSI bloodstream infection 
Table 1 Baseline characteristics and clinical outcomes of patients with methicillin-susceptible S. aureus bacteremia. Patients received empiric antimicrobial therapy with either $\beta$-lactams or vancomycin

\begin{tabular}{|c|c|c|c|}
\hline Patient characteristics & $\beta$-lactam ${ }^{a}(n=200)$ & Vancomycin $^{\mathrm{a}}(n=200)$ & $P$-value \\
\hline $\mathrm{Age}^{\mathrm{b}}$ & $53.0 \pm 16.9$ & $57.9 \pm 18.4$ & 0.01 \\
\hline Males & $126(63.0)$ & $132(66.0)$ & 0.53 \\
\hline Community-onset & $173(86.5)$ & $158(79.0)$ & 0.05 \\
\hline Hospital-onset & $27(13.5)$ & $42(21.0)$ & 0.05 \\
\hline HIV infection & $20(10.0)$ & $16(8.0)$ & 0.48 \\
\hline Hepatitis C infection & $61(30.5)$ & $55(27.5)$ & 0.51 \\
\hline Immunocompromised & $18(9.0)$ & $16(8.0)$ & 0.72 \\
\hline Alcohol or illicit drug abuse & $82(41.0)$ & $71(35.5)$ & 0.26 \\
\hline Intravenous drug use & $64(32.0)$ & $56(28.0)$ & 0.38 \\
\hline Charlson comorbidity index ${ }^{c}$ & $3(1.0-6.0)$ & $4(1.0-7.0)$ & 0.01 \\
\hline Pitt bacteremia score ${ }^{c}$ & $1(0-2)$ & $1(0-2)$ & 0.18 \\
\hline Infectious diseases consultation & $150(75.0)$ & $132(66.0)$ & 0.05 \\
\hline \multicolumn{4}{|l|}{ Source of bacteremia } \\
\hline Central or peripheral line & $24(12.0)$ & $37(18.5)$ & 0.09 \\
\hline Skin and soft tissue & $36(18.0)$ & $24(12.0)$ & 0.12 \\
\hline Intravenous drug use & $48(24.0)$ & $39(19.5)$ & 0.33 \\
\hline Bone or joint infection & $23(11.5)$ & $13(6.5)$ & 0.11 \\
\hline Lung & $6(3.0)$ & $7(3.5)$ & 1.00 \\
\hline Other & $19(9.5)$ & $20(10.0)$ & 1.00 \\
\hline Unknown & $44(22.0)$ & $60(30.0)$ & 0.09 \\
\hline Infective endocarditis & $45(22.5)$ & $23(11.5)$ & $<0.01$ \\
\hline Metastatic complications & $73(36.5)$ & $53(26.5)$ & 0.03 \\
\hline Surgical source control & $43(21.5)$ & $26(13.0)$ & 0.02 \\
\hline \multicolumn{4}{|l|}{ Empiric antimicrobials } \\
\hline$\beta$-lactam & $200(100)$ & $75(37.5)$ & $<0.0001$ \\
\hline Cloxacillin or cefazolin & $138(69.0)$ & $10(5.0)$ & $<0.0001$ \\
\hline $3^{\text {rd }}$ generation cephalosporin & $80(40.0)$ & $35(17.5)$ & $<0.0001$ \\
\hline Piperacillin-tazobactam & $69(34.5)$ & $35(17.5)$ & $<0.001$ \\
\hline Ticarcillin-clavulanic acid & $3(1.5)$ & $1(0.5)$ & 0.62 \\
\hline Carbapenem & $8(4.0)$ & $4(2.0)$ & 0.24 \\
\hline Vancomycin & $153(76.5)$ & $197(98.5)$ & $<0.0001$ \\
\hline Daptomycin & $2(1.0)$ & $1(0.5)$ & 1.00 \\
\hline Linezolid & $2(1.0)$ & $2(1.0)$ & 1.00 \\
\hline Other $^{d}$ & $82(41.0)$ & $97(48.5)$ & 0.13 \\
\hline Blood culture time to positivity ${ }^{e}$ & $20.2(16.6-25.5)$ & $18.5(16.2-23.3)$ & 0.02 \\
\hline Duration of empiric therapy ${ }^{e}$ & $55.4(44.2-72.5)$ & $52.1(39.2-75.7)$ & 0.55 \\
\hline Duration of definitive therapy ${ }^{f}$ & $28(13.0-42.0)$ & $26.5(11.0-42.0)$ & 0.14 \\
\hline Time to receipt of empiric therapy ${ }^{e}$ & $1.92(0.1-6.9)$ & $10.8(1.4-24.1)$ & $<0.0001$ \\
\hline Time to receipt of $\beta$-lactam ${ }^{e}$ & $2.92(0.3-13.8)$ & $50.5(4.8-75.5)$ & $<0.0001$ \\
\hline Time to receipt of cloxacillin or cefazoline & $31.0(13.8-50.8)$ & $67.8(50.3-88.0)$ & $<0.0001$ \\
\hline Empiric $\beta$-lactam exposure & $\begin{array}{l}52.3(39.8-71.9)^{\mathrm{e}} \\
60.6 \pm 39.9^{\mathrm{g}}\end{array}$ & $\begin{array}{l}0(0-16.2)^{\mathrm{e}} \\
9.5 \pm 38.5^{\mathrm{g}}\end{array}$ & $<0.0001$ \\
\hline Proportional empiric $\beta$-lactam exposure & $\begin{array}{l}100(100-100)^{h} \\
94.5 \pm 46.9^{i}\end{array}$ & $\begin{array}{l}0(0-24.5)^{\mathrm{h}} \\
14.0 \pm 44.8^{\mathrm{i}}\end{array}$ & $<0.0001$ \\
\hline
\end{tabular}


Table 1 Baseline characteristics and clinical outcomes of patients with methicillin-susceptible S. aureus bacteremia. Patients received empiric antimicrobial therapy with either $\beta$-lactams or vancomycin (Continued)

\begin{tabular}{|c|c|c|c|}
\hline \multicolumn{4}{|l|}{ Primary outcome } \\
\hline 28-day mortality & $16(8.0)$ & $18(9.0)$ & 0.72 \\
\hline \multicolumn{4}{|l|}{ Secondary outcomes } \\
\hline 90-day mortality & $25(12.5)$ & $32(16.0)$ & 0.32 \\
\hline Recurrent infection at 6 months & $7(3.5)$ & $8(4.0)$ & 0.79 \\
\hline Duration of bacteremia ${ }^{\mathrm{e}, j}$ & $74.4(48.3-130)$ & $89.7(56.7-132)$ & 0.20 \\
\hline$\geq 3$ days $^{j}$ & $98(53.8)$ & $111(60.0)$ & 0.25 \\
\hline Hospital length of stay ${ }^{f}$ & $22.5(12.5-43.0)$ & $22(13.0-45.0)$ & 0.59 \\
\hline 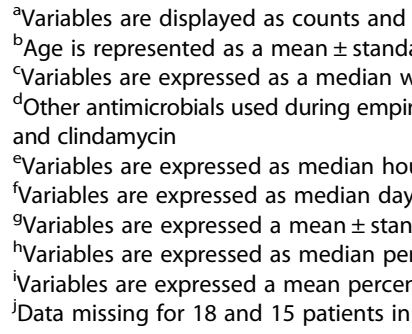 & $\begin{array}{l}\text { s unless otherwise } \\
\text { cludentheses } \\
\text { rifampin, am } \\
\text { in parentheses } \\
\text { in parentheses } \\
\text { e range in parenthe } \\
\text { ycin group, respecti }\end{array}$ & inolones, macrolide & xazole \\
\hline
\end{tabular}

34.8 vs $13.0 \mathrm{~h}, p=0.00)$. The combination subgroup had a higher Pitt bacteremia score (median 1 vs $0, p<0.01$ ), received more infectious diseases consultations $(78.4 \%$ vs $63.8 \%, p=0.05)$, and experienced more metastatic complications $(43.1 \%$ vs $14.9 \%, p<0.001)$ than the $\beta$ lactam monotherapy subgroup. Both the duration of bacteremia (median 84.8 vs $63.4 \mathrm{~h}, p=0.03$ ) and hospital LOS (median 26 vs 15 days, $p<0.01$ ) were longer in the combination subgroup.

Almost half of patients in the vancomycin group received additional antimicrobials during empiric or definitive therapy (Table 1). Rifampin and aminoglycosides were added for synergy in patients with prosthetic valve infective endocarditis or prosthetic joint infections. Fluoroquinolones, macrolides, trimethoprim-sulfamethoxazole, and clindamycin were used for either treatment of nonbacteremic co-infections or prophylaxis for other medical conditions. Three patients in the vancomycin group received an incomplete dose of vancomycin and were counted as not having received it.

There was a greater delay in receipt of empiric antimicrobials in the vancomycin group compared to the $\beta$-lactam group (median 10.8 vs $1.9 \mathrm{~h}, p<0.0001$ ) (Table 1$)$. Seventy five $(37.5 \%)$ patients in the vancomycin group were briefly exposed to $\beta$-lactams during empiric therapy. However, exposure time (median 0 vs $52.3 \mathrm{~h}, p<0.0001$ ) and proportional time of exposure of the empiric period (median 0 vs $100 \%, p<0.0001$ ) were miniscule compared to the $\beta$-lactam group. There were no differences in clinical outcomes between the two groups (Table 3). The overall 28-day and 90-day mortality was 34 (8.5\%) and 57 (14.3\%) respectively. Among patients with infective endocarditis, 28-day and 90-day mortality was $4(8.89 \%)$ and $7(15.6 \%)$ in the $\beta$ lactam group and 0 and $3(13.0 \%)$ in the vancomycin group. In the vancomycin group, the subset of patients who received brief exposure to empiric $\beta$-lactam experienced faster clearance of bacteremia compared to those who did not have any empiric $\beta$-lactam exposure (median 78.9 vs $96.3 \mathrm{~h}, p=0.04$ ). In comparison with the $\beta$-lactam group, patients in the vancomycin group who did not have any empiric $\beta$-lactam exposure had slightly higher 90-day mortality [22 (17.6 \%) vs 25 (12.5\%), $p=0.01$ ] and longer duration of bacteremia (median $96.3 \mathrm{vs} 74.4 \mathrm{~h}$, $p=0.03$ ), while those who were briefly exposed to empiric $\beta$-lactams exhibited no difference in clinical outcomes.

\section{Discussion}

The goal of our study was to assess if empiric $\beta$-lactams compared to vancomycin was associated with differences in outcomes in patients with MSSA bacteremia. We found no differences in all-cause mortality at 28 and 90 days, recurrent infection at 6 months, duration of bacteremia or hospital LOS between patients treated with empiric $\beta$-lactam or vancomycin therapy. However, in the vancomycin group, the subset of patients who were not exposed to any empiric $\beta$-lactams had higher 90-day mortality and longer duration of bacteremia, while those who had even minimal exposure to empiric $\beta$-lactams did not have worse outcomes. Patients in the vancomycin group were older, had more medical comorbidities, were less likely to be assessed by an infectious diseases consultant, underwent fewer source control procedures, and experienced a greater delay in receipt of empiric antibiotics compared to the $\beta$-lactam group. 
Table 2 Baseline characteristics and clinical outcomes of patients with methicillin-susceptible S. aureus bacteremia. Patients received either empiric combination therapy with $\beta$-lactam plus vancomycin or empiric $\beta$-lactam monotherapy

\begin{tabular}{|c|c|c|c|}
\hline Patient characteristics & $\beta$-lactam plus vancomycin ${ }^{a}(n=153)$ & $\beta$-lactam monotherapy ${ }^{\mathrm{a}}(n=47)$ & $P$-value \\
\hline $\mathrm{Age}^{\mathrm{b}}$ & $51.3 \pm 16.9$ & $58.7 \pm 16.1$ & $<0.01$ \\
\hline Males & $92(60.1)$ & $34(72.3)$ & 0.17 \\
\hline Community-onset & $137(89.5)$ & $36(76.6)$ & 0.03 \\
\hline Hospital-onset & $16(10.5)$ & $11(23.4)$ & 0.03 \\
\hline HIV infection & $18(11.8)$ & $2(4.26)$ & 0.17 \\
\hline Hepatitis C infection & $50(32.7)$ & $11(23.4)$ & 0.28 \\
\hline Immunocompromised & $12(7.84)$ & $6(12.8)$ & 0.38 \\
\hline Alcohol or illicit drug abuse & $68(44.4)$ & $14(29.8)$ & 0.09 \\
\hline Intravenous drug use & $54(35.3)$ & $10(21.3)$ & 0.08 \\
\hline Charlson comorbidity index ${ }^{c}$ & $3(1-6)$ & $3(1-5)$ & 0.30 \\
\hline Pitt bacteremia score ${ }^{c}$ & $1(0-2)$ & $0(0-1)$ & $<0.01$ \\
\hline Infectious diseases consultation & $120(78.4)$ & $30(63.8)$ & 0.05 \\
\hline \multicolumn{4}{|l|}{ Source of bacteremia } \\
\hline Central or peripheral line & $15(9.80)$ & $9(19.1)$ & 0.12 \\
\hline Skin and soft tissue & $25(16.3)$ & $11(23.4)$ & 0.28 \\
\hline Intravenous drug use & $43(28.1)$ & $5(10.6)$ & 0.02 \\
\hline Bone or joint infection & $15(9.80)$ & $8(17.0)$ & 0.19 \\
\hline Lung & $4(2.61)$ & $2(4.26)$ & 0.63 \\
\hline Other & $14(9.15)$ & $5(10.6)$ & 0.78 \\
\hline Unknown & $37(24.2)$ & $7(14.9)$ & 0.23 \\
\hline Infective endocarditis & $40(26.1)$ & $5(10.6)$ & 0.03 \\
\hline Metastatic complications & $66(43.1)$ & $7(14.9)$ & $<0.001$ \\
\hline Surgical source control & $31(20.3)$ & $12(25.5)$ & 0.43 \\
\hline \multicolumn{4}{|l|}{ Empiric antimicrobials } \\
\hline Cloxacillin or cefazolin & $95(62.1)$ & $43(91.5)$ & $<0.0001$ \\
\hline $3^{\text {rd }}$ generation cephalosporin & $73(47.7)$ & $7(14.9)$ & $<0.0001$ \\
\hline Piperacillin-tazobactam & $65(42.5)$ & $4(8.51)$ & $<0.0001$ \\
\hline Ticarcillin-clavulanic acid & $2(1.31)$ & $1(2.13)$ & 0.55 \\
\hline Carbapenem & $7(4.58)$ & $1(2.13)$ & 0.68 \\
\hline Daptomycin & 0 & $2(4.26)$ & 0.05 \\
\hline Linezolid & $1(0.65)$ & $1(2.13)$ & 0.42 \\
\hline Other $^{d}$ & $60(39.2)$ & $22(46.8)$ & 1.00 \\
\hline Blood culture time to positivity ${ }^{e}$ & $20.3(16.3-25.3)$ & $19.8(18.0-27)$ & 0.36 \\
\hline Duration of empiric therapy ${ }^{e}$ & $55.5(44.9-73.8)$ & $54.3(38.8-64.5)$ & 0.08 \\
\hline Duration of definitive therapy ${ }^{f}$ & $31(14-43)$ & $26(12-40)$ & 0.18 \\
\hline Time to receipt of empiric therapy ${ }^{e}$ & $1.6(0.03-6.17)$ & $3.95(0.58-15.5)$ & 0.08 \\
\hline Time to receipt of $\beta$-lactam ${ }^{\mathrm{e}}$ & $2.77(0.25-13.7)$ & $3.95(0.58-16.6)$ & 0.74 \\
\hline Time to receipt of cloxacillin or cefazolin ${ }^{e}$ & $34.8(21.2-58.4)$ & $13.0(1.83-23.8)$ & 0.00 \\
\hline Empiric $\beta$-lactam exposure ${ }^{\mathrm{e}}$ & $52.3(39.8-73.8)$ & $51.7(38.8-64.5)$ & 0.42 \\
\hline Proportional empiric $\beta$-lactam exposure ${ }^{9}$ & $100(86.1-100)$ & $100(95.2-100)$ & 0.01 \\
\hline \multicolumn{4}{|l|}{ Primary outcome } \\
\hline 28-day mortality & $14(9.15)$ & $2(4.26)$ & 0.37 \\
\hline
\end{tabular}


Table 2 Baseline characteristics and clinical outcomes of patients with methicillin-susceptible S. aureus bacteremia. Patients received either empiric combination therapy with $\beta$-lactam plus vancomycin or empiric $\beta$-lactam monotherapy (Continued)

\begin{tabular}{|c|c|c|c|}
\hline \multicolumn{4}{|l|}{ Secondary outcomes } \\
\hline 90-day mortality & $21(13.7)$ & $4(8.51)$ & 0.45 \\
\hline Recurrent infection at 6 months & $5(3.27)$ & $2(4.26)$ & 0.67 \\
\hline Duration of bacteremia ${ }^{\mathrm{e}, \mathrm{h}}$ & $84.8(52.5-136)$ & $63.4(30.5-114)$ & 0.03 \\
\hline$\geq 3$ days $^{h}$ & $81(56.3)$ & $17(44.7)$ & 0.27 \\
\hline Hospital length of stay ${ }^{f}$ & $26(13-45)$ & $15(10-30)$ & $<0.01$ \\
\hline $\begin{array}{l}\text { a Variables are displayed as counts and } \\
{ }^{b} \text { Age is represented as a mean } \pm \text { stand } \\
{ }^{c} \text { Variables are expressed as a median } \\
{ }^{\mathrm{d}} \text { Other antimicrobials used during empir } \\
\text { and clindamycin } \\
{ }^{\mathrm{e}} \text { Variables are expressed as median ho } \\
{ }^{\mathrm{f}} \text { Variables are expressed as median day } \\
{ }^{\mathrm{g}} \text { Variables are expressed as median per } \\
{ }^{\mathrm{h}} \text { Data missing for } 9 \text { patients in each su }\end{array}$ & $\begin{array}{l}\text { arentheses unless } \\
\text { years } \\
\text { range in parenthes } \\
\text { therapy included rif } \\
\text { rtile range in paren } \\
\text { tile range in parenth } \\
\text { terquartile range in }\end{array}$ & inolones, macrolide & azole, \\
\hline
\end{tabular}

More cases of infective endocarditis and metastatic complications were diagnosed $\beta$-lactam group. Despite the high prevalence of MRSA at both of our institutions ( $25 \%$ and $38 \%$ ), only $76.5 \%$ of patients in the $\beta$-lactam group received vancomycin empirically as well. Perhaps the awareness of MRSA was low among some treating clinicians or patients who did not receive empiric vancomycin were judged to be at low risk for MRSA infection. Although the combination of $\beta$-lactams with vancomycin exhibits synergistic killing against MRSA, neither synergy nor antagonism was observed against MSSA in vitro [21]. Therefore, the addition of vancomycin to $\beta$ lactams would not be expected to influence microbiological cure in MSSA bacteremia.

Interestingly, the differential time delay in receipt of empiric antimicrobials was unexpected. The $\beta$-lactam group received antimicrobial therapy earlier possibly because these patients were more severely ill as reflected in their higher rate of infective endocarditis and metastatic complications, despite similar Pitt bacteremia scores between the two groups. Infectious diseases consultation may have also contributed to earlier initiation of antibiotics in the $\beta$-lactam group.
In the $\beta$-lactam group, patients who received empiric $\beta$-lactam plus vancomycin were generally sicker as indicated by their higher Pitt bacteremia score, higher rate of infective endocarditis and metastatic complications, and longer duration of bacteremia and hospital LOS, compared to those who received empiric $\beta$-lactam monotherapy. The greater severity of illness in this combination subgroup may explain the initial use of broad-spectrum $\beta$-lactams (ceftriaxone or piperacillintazobactam), with subsequent de-escalation to cloxacillin or cefazolin in some patients during the empiric period by the infectious diseases consultant when $S$. aureus was identified in the blood culture. De-escalation occurred within a median of $34.8 \mathrm{~h}$, which follows the time to positivity of the first blood culture (median $20.3 \mathrm{~h}$ ).

The 28-day and 90-day mortality in our study was low at $34(8.5 \%)$ and $57(14.3 \%)$ respectively, but is within the range of 3.6 to $51.7 \%$ reported in a meta-analysis of patients with MSSA bacteremia from catheter-related infections and infective endocarditis by Cosgrove et al. [22]. Definite infective endocarditis was diagnosed in 68 (17\%) of our patients, which is similar to rates reported in previous studies $[10,14,16,23]$.

Table 3 Outcome analysis comparing $\beta$-lactam versus vancomycin group. Variables were adjusted for predefined confounding variables, including age, sex, age-adjusted Charlson-comorbidity index, Pitt bacteremia score, infectious diseases consultation, infective endocarditis and time to receipt of empiric antibiotics. Duration of bacteremia was further adjusted for surgical source control

\begin{tabular}{lllll}
\hline Outcomes & Crude OR $(95 \% \mathrm{Cl})$ & $P$-value & Adjusted OR (95\% Cl) & $P$-value \\
28-day mortality & $0.88(0.44-1.78)$ & 0.72 & $1.14(0.49-2.64)$ & 0.76 \\
90-day mortality & $0.75(0.43-1.32)$ & 0.32 & $1.01(0.51-2.02)$ & 0.97 \\
Recurrent infection at 6 months & $0.87(0.31-2.45)$ & 0.79 & $1.27(0.39-4.11)$ & 0.69 \\
& Ratio of Mean $(95 \% \mathrm{Cl})$ & $P$-value & Adjusted Ratio of Mean $(95 \% \mathrm{Cl})$ & $P$-value \\
Duration of bacteremia & $0.92(0.78-1.07)$ & 0.27 & $0.94(0.79-1.11)$ & 0.44 \\
Hospital length-of-stay & $0.96(0.81-1.14)$ & 0.65 & $0.95(0.80-1.14)$ & 0.60 \\
\hline
\end{tabular}

Abbreviations: $O R$ odds ratio, $\mathrm{Cl}$ confidence interval 
In the vancomycin group, the median time to definitive treatment with cloxacillin or cefazolin was $67.8 \mathrm{~h}$, which is comparable to other studies $[3,16,17]$. In the Khatib study, a delayed clearance of bacteremia ( $\geq 3$ days) was observed in the vancomycin group compared to the $\beta$-lactam group ( $57.6 \%$ vs $37.5 \%$ ). However, there were no differences in all-cause or attributable mortality between groups. Among injection drug users with predominantly right-sided MSSA infective endocarditis, Lodise et al. demonstrated that infection-related mortality was lower with empiric $\beta$-lactam than with vancomycin monotherapy (11.4 \% vs $39.3 \%, p=0.005)$ [17]. Even when patients were switched from vancomycin to a semi-synthetic penicillin within a median of 3 days, infection-related mortality remained high at $40.9 \%$. The overall mortality in this cohort was unusually high at $22.2 \%$ compared to a rate of $0-4 \%$ described in a systematic review by Yung et al. [24]. In contrast, a recent study by McDanel et al. found that empiric $\beta$-lactam therapy (predominantly piperacillin-tazobactam and ceftriaxone) compared to vancomycin was not associated with differences in mortality in patients with MSSA bacteremia [15]. However, the McDanel study excluded patients who received empiric vancomcyin plus $\beta$ lactams, and clinical outcomes were evaluated independent of the antimicrobial prescribed for definitive therapy.

At institutions where MRSA prevalence is significant, vancomycin is generally accepted as an appropriate empiric antimicrobial for $S A B$, but due to conflicting results from prior studies, the addition of a $\beta$-lactam agent to empiric therapy remains controversial. $\beta$-lactam monotherapy may be inadequate empiric treatment if the strain is methicillin-resistant, but mortality outcomes from retrospective studies are mixed [3, 6, 8, 25]. Ultimately, the choice of empiric therapy will depend on patient factors, the prevalence of MRSA in the population, and the ability of the microbiology laboratory to rapidly differentiate MSSA from MRSA. From the perspective of antimicrobial stewardship, vancomycin monotherapy seems favourable as the reduction in usage of $\beta$-lactams may decrease the potential for drug-drug interactions and adverse effects during empiric therapy for SAB.

A particular strength of our study is the inclusion of a large proportion of patients who received optimal anti-MSSA agents (cloxacillin and cefazolin) empirically. The major limitation of previous studies was the lack of transparency regarding the empiric $\beta$-lactams prescribed $[3,17]$. This is important because not all $\beta$ lactams have the same activity against MSSA. In one retrospective study, second and third generation cephalosporins and $\beta$-lactam $/ \beta$-lactamase inhibitor combinations were inferior to cloxacillin and cefazolin for empiric treatment of MSSA bacteremia [26]. We did not perform subgroup analysis for different $\beta$-lactams because antimicrobials were frequently switched during empiric therapy.

Our study has several limitations. The reason for the lack of difference in the primary outcome is likely multifactorial. Because of the low event rate in both groups, our study was potentially underpowered to detect a significant difference in mortality. The lower than expected death rate may be partly due to the exclusion of patients who died within $24 \mathrm{~h}$ of the diagnosis of SAB and of patients who remained on broad-spectrum $\beta$-lactams. This group may have represented a sicker population and thus, we may have selected for less critically ill patients. Due to the retrospective nature of this study, baseline characteristics between the two groups were significantly different, although we did attempt to control for these differences in the multivariable model. Future studies will need to employ matching strategies to eliminate this imbalance. Although there appears to be a protective effect of even brief exposure to empiric $\beta$-lactams, caution must be exercised when interpreting this data due to the small sample size and major differences in baseline characteristics between treatment groups. Because the source of bacteremia was not identified in a significant proportion of patients in both groups, clinical outcomes may have been impacted by a lack of source control. As two different microbiology laboratories were involved in the study, differences in the detection methods of MSSA may have affected the timing of definitive therapy. Obtaining subsequent blood cultures was often delayed or sometimes not performed at all, which may have led to an overestimation of the duration of bacteremia in both groups. Data regarding adverse effects were not collected due to the inherent difficulty of establishing drug-related events in a retrospective study. We were not able to determine if patients received appropriate dosing of antibiotics because data on antibiotic doses and vancomycin trough levels were not collected. A randomized controlled trial would be needed to confirm our study findings.

\section{Conclusions}

Empiric therapy with $\beta$-lactams was not associated with differences in all-cause mortality, recurrent infection, microbiological cure or hospital LOS compared to vancomycin in patients with MSSA bacteremia. Vancomycin monotherapy may be appropriate for the empiric treatment of MSSA bacteremia if definitive therapy with cloxacillin or cefazolin can be initiated within 3 days.

\section{Abbreviations}

BSI: bloodstream infection; LOS: length-of-stay; MRSA: methicillin-resistant Staphylococcus aureus; MSSA: methicillin-susceptible Staphylococcus aureus; SAB: Staphylococcus aureus bacteremia. 


\section{Acknowledgements}

We thank the members of Centre for Health Evaluation \& Outcome Sciences, Dr. Joel Singer, Rachel McKay, Joseph Puyat and Hong Qian, for their assistance with the statistical analyses.

\section{Funding}

This study was not supported by external funding.

\section{Availability of data and materials}

Data supporting the findings are included in the manuscript.

\section{Authors' contributions}

DW conceived and designed the study, collected and analyzed the data, drafted and revised the manuscript. TW participated in study design and revised the manuscript. MR made critical revisions to the manuscript. $V L$ participated in study design, interpreted the data and revised the manuscript. All authors read and approved the final manuscript.

\section{Competing interests}

The authors declare that they have no competing interests.

\section{Consent to publish}

Not applicable.

\section{Ethics and consent to participate}

The study was approved by the research ethics board at the University of British Columbia with waiver of informed consent, and received institutional approval from Vancouver Coastal Health and Providence Healthcare.

\section{Author details}

'PGY-V Infectious Diseases Residency Training Program, University of British Columbia, Vancouver General Hospital, D 452 Heather Pavilion, 2733 Heather Street, Vancouver, BC V5Z 1 M9, Canada. ${ }^{2}$ Department of Pathology and Laboratory Medicine, University of British Columbia, Vancouver, BC, Canada. ${ }^{3}$ Division of Medical Microbiology and Infection Control, Vancouver General Hospital, JPPN1, Medical Microbiology Laboratory, 899 W 12th Ave, Vancouver, BC V5Z 1 M9, Canada. ${ }^{4}$ Division of Medical Microbiology, St. Paul's Hospital, Medical Microbiology Laboratory, 1081 Burrard St., Vancouver, BC V6Z 1Y6, Canada.

\section{Received: 26 November 2015 Accepted: 12 May 2016} Published online: 23 May 2016

\section{References}

1. Van Hal SJ, Jensen SO, Vaska VL, Espedido BJ, Paterson DL, Gosbell IB. Predictors of mortality in Staphylococcus aureus bacteremia. Clin Microbiol Rev. 2012;25:362-86

2. Thwaites GE, Edgeworth JD, Gkrania-Klotsas E, Kirby A, Tilley R, Torok ME, et al. Clinical management of Staphylococcus aureus bacteraemia. Lancet Infect Dis. 2011;11:208-22.

3. Khatib R, Saeed S, Sharma M, Riederer K, Fakih MG, Johnson LB. Impact of initial antibiotic choice and delayed appropriate treatment on the outcome of Staphylococcus aureus bacteremia. Eur J Clin Microbiol Infect Dis. 2006:25:181-5.

4. Paul M, Kariv G, Goldberg E, Raskin M, Shaked H, Hazzan R, et al. Importance of appropriate empirical antibiotic therapy for methicillin-resistant Staphylococcus aureus bacteraemia. J Antimicrob Chemother. 2010;65:2658-65.

5. David MZ, Daum RS. Community-associated methicillin-resistant Staphylococcus aureus: epidemiology and clinical consequences of an emerging epidemic. Clin Microbiol Rev. 2010;23:616-87.

6. Lodise TP, McKinnon PS, Swiderski L, Rybak M. Outcomes analysis of delayed antibiotic treatment for hospital-acquired Staphylococcus aureus bacteremia. Clin Infect Dis. 2003:36:1418-23.

7. Marchaim D, Kaye KS, Fowler VG, Anderson DJ, Chawla V, Golan Y. Casecontrol study to identify factors associated with mortality among patients with methicillin-resistant Staphylococcus aureus bacteraemia. Clin Microbio Infect. 2010;16:747-52.

8. Schweizer ML, Furuno JP, Harris AD, Johnson JK, Shardell MD, McGregor JC Empiric antibiotic therapy for Staphylococcus aureus bacteremia May Not reduce in-hospital mortality: a retrospective cohort study. PLoS One. 2010;5:e11432.
9. Kim SH, Kim KH, Kim HB, Kim NJ, Kim EC, Oh MD, et al. Outcome of vancomycin treatment in patients with methicillin-susceptible Staphylococcus aureus bacteremia. Antimicrob Agents Chemother. 2008;52:192-7.

10. Lee S, Choe PG, Song KH, Park SW, Kim HB, Kim NJ, et al. Is cefazolin inferior to nafcillin for treatment of methicillin-susceptible Staphylococcus aureus bacteremia? Antimicrob Agents Chemother. 2011;55:5122-6.

11. Liu C, Bayer A, Cosgrove SE, Daum RS, Fridkin SK, Gorwitz RJ, et al. Clinical practice guidelines by the infectious diseases society of America for the treatment of methicillin-resistant Staphylococcus aureus infections in adults and children. Clin Infect Dis. 2011;52:1-38

12. Chang FY, Peacock JE, Musher Jr DM, Triplett P, MacDonald BB, Mylotte JM, et al. Staphylococcus aureus bacteremia recurrence and the impact of antibiotic treatment in a prospective multicenter study. Medicine. 2003;82:333-9.

13. Chan KE, Warren HS, Thadhani RI, Steele DJR, Hymes JL, Maddux FW, et al. Prevalence and outcomes of antimicrobial treatment for Staphylococcus aureus bacteremia in outpatients with ESRD. J Am Soc Nephrol. 2012;23:1551-9.

14. Stryjewski ME, Szczech LA, Benjamin Jr DK, Inrig JK, Kanafani ZA, Engemann $J$ J, et al. Use of vancomycin or first-generation cephalosporins for the treatment of hemodialysis-dependent patients with MSSA bacteremia. Clin Infect Dis. 2007:44:190-6.

15. McDanel JS, Perencevich EN, Diekema DJ, Herwaldt LA, Smith TC, Chrischilles EA, et al. Comparative effectiveness of beta-lactams versus vancomycin for treatment of methicillin-susceptible Staphylococcus aureus bloodstream infections among 122 hospitals. Clin Infect Dis. 2015:61:361-7.

16. Schweizer ML, Furuno JP, Harris AD, Johnson JK, Shardell MD, McGregor JC, et al. Comparative effectiveness of nafcillin or cefazolin versus vancomycin in methicillin-susceptible Staphylococcus aureus bacteremia. BMC Infect Dis. 2011;11:279.

17. Lodise Jr TP, McKinnon PS, Levine DP, Rybak MJ. Impact of empirical-therapy selection on outcomes of intravenous drug users with infective endocarditis caused by MSSA. Antimicrob Agents Chemother. 2007:51:3731-3.

18. McConeghy KW, Bleasdale SC, Rodvold KA. The empirical combination of vancomycin and a $\beta$-lactam for staphylococcal bacteremia. Clin Infect Dis. 2013;57:1760-5

19. Li JS, Sexton DJ, Mick N, Nettles R, Fowler Jr VG, Ryan T, et al. Proposed modifications to the duke criteria for the diagnosis of infective endocarditis. Clin Infect Dis. 2000;30:633-8.

20. Schneeweiss S, Wang PS, Avorn J, Glynn RJ. Improved comorbidity adjustment for predicting mortality in medicare populations. Health Serv Res. 2003;38:1103-20.

21. Joukhadar C, Pillai S, Wennersten C, Moellering RC, Eliopoulos Jr GM. Lack of bactericidal antagonism or synergism in vitro between oxacillin and vancomycin against methicillin-susceptible strains of Staphylococcus aureus. Antimicrob Agents Chemother. 2010:54:773-7.

22. Cosgrove SE, Sakoulas G, Perencevich EN, Schwaber MJ, Karchmer AW, Carmeli Y. Comparison of mortality associated with methicillin-resistant and methicillin-susceptible Staphylococcus aureus bacteremia: a meta-analysis. Clin Infect Dis. 2003:36:53-9.

23. Bai AD, Showler A, Burry L, Steinberg M, Ricciuto DR, Fernandes T, et al. Comparative effectiveness of cefazolin versus cloxacillin as definitive antibiotic therapy for MSSA bacteraemia: results from a large multicentre cohort study. J Antimicrob Chemother. 2015;70:1539-46.

24. Yung D, Kottachchi D, Neupane B, Haider S, Loeb M. Antimicrobials for right-sided endocarditis in intravenous drug users: a systematic review. Antimicrob Chemother. 2007:60:921-8.

25. Rodriguez-Bano J, Millan AB, Dominguez MA, Borraz C, Gonzalez MP, Almirante $B$, et al. Impact of inappropriate empirical therapy for sepsis due to health care-associated methicillin-resistant Staphylococcus aureus. J Infect. 2009;58:131-7.

26. Paul M, Zemer-Wassercug N, Talker O, Lishtzinsky Y, Lev B, Samra Z, et al. Are all beta-lactams similarly effective in the treatment of methicillin-sensitive Staphylococcus aureus bacteraemia. Clin Microbiol Infect. 2011;17:1581-6. 